\title{
Influence of apolipoprotein $E$ plasma levels and tobacco smoking on the induction of neutralising antibodies to interferon-beta
}

\author{
Armando Sena $\cdot$ Klaus Bendtzen - Maria J. Cascais • \\ Rui Pedrosa $\cdot$ Véronique Ferret-Sena $\cdot$ Elisa Campos
}

Received: 15 February 2010/Revised: 15 May 2010/Accepted: 17 May 2010/Published online: 4 June 2010

(c) Springer-Verlag 2010

\begin{abstract}
Interferon-beta (IFN-beta) therapy for multiple sclerosis (MS) is associated with a potential for induction of neutralizing antibodies (NAbs). Because immune reactivity depends on changes in lipoprotein metabolism, we investigated whether plasma lipoprotein profiles could be associated with the development of NAbs. Thirty-one female MS patients treated with subcutaneously administered IFN-beta were included. Demographic and clinical characteristics were compared between NAbs response groups using $t$ tests for continuous and logistic regression analysis and Fisher's exact tests for categorical data, respectively. Multivariate logistic regression was used to evaluate the effect of potential confounders. Patients who developed NAbs had lower apoE
\end{abstract}

A. Sena $(\bowtie) \cdot$ M. J. Cascais · E. Campos

CEDOC, Departamento de Bioquímica, Faculdade de Ciências

Médicas, Universidade Nova de Lisboa, Lisbon, Portugal

e-mail: armando.sena@fcm.unl.pt

M. J. Cascais

e-mail: cascaismaria@hotmail.com

E. Campos

e-mail: elisamscampos@gmail.com

A. Sena $\cdot$ R. Pedrosa

Departamento de Neurociências, Centro Hospitalar de

Lisboa-Central, Lisbon, Portugal

R. Pedrosa

e-mail: ruipedrosa@ netcabo.pt

\section{K. Bendtzen}

Institute for Inflammation Research IIR7521, Rigshospitalet

National University Hospital, Copenhagen, Denmark

e-mail: kben@dbmail.dk

\section{Ferret-Sena}

Departamento de Fisiologia, Centro de Investigação Interdisciplinar Egas Moniz, Monte de Caparica, Portugal

e-mail: versena@egasmoniz.edu.pt levels before treatment, 67 (47-74) $\mathrm{mg} / \mathrm{L}$ median (interquartile range), and at the moment of NAb analysis, 53 (50-84) $\mathrm{mg} / \mathrm{L}$, in comparison to those who remained NAb-negative, 83 (68-107) $\mathrm{mg} / \mathrm{L}, P=0.03$, and 76 (66-87) $\mathrm{mg} / \mathrm{L}, P=0.04$, respectively. When adjusting for age and smoking for a onestandard deviation decrease in apoE levels, a 5.6-fold increase in the odds of becoming NAb-positive was detected: odds ratios (OR) 0.18 (95\% CI 0.04-0.77), $P=0.04$. When adjusting for apoE, smoking habit became associated with NAb induction: OR 5.6 (95\% CI 1.3-87), $P=0.03$. These results suggest that apoE-containing lipoprotein metabolism and, possibly, tobacco smoking may be associated with risk of NAb production in female MS patients treated with IFN-beta.

Keywords Multiple sclerosis - Interferon-beta . Neutralising antibodies - Lipoproteins - Apolipoprotein E . Tobacco smoking

\section{Introduction}

Interferon-beta (IFN-beta) therapy for multiple sclerosis (MS) is associated with a potential for development of neutralising antibodies (NAbs). Several recent studies have shown that the clinical efficacy of IFN-beta is diminished by the presence of NAbs [1, 2]. Differences related to IFNbeta preparations, dose and frequency of administration and individual genetic factors are known to affect the risk for NAbs production [3, 4]. However, metabolic characteristics also influence immune responses, and hence might affect NAbs development in MS patients. For example, recent data have emphasized the relationships between lipoprotein metabolism and both innate and adaptive immunity [5, 6]; alterations in plasma lipoproteins levels 
have also been found in MS patients [7, 8]. However, the pathogenic and clinical importance of these alterations in MS remains uncertain. We therefore investigated whether the plasma lipoprotein profiles of MS patients treated with IFN-beta were associated with risk of developing NAbs. Patients with and without NAbs were further characterized by assessments of neopterin, ferritin, leptin and uric acid plasma levels. The potential influence of other medications and tobacco smoking was also investigated.

\section{Methods}

\section{Patients}

Thirty-one female relapsing-remitting MS patients, according the McDonald criteria [9], who initiated IFNbeta therapy were included in this study. These patients were recruited from the MS outpatient clinic at the Department of Neurosciences (Neurology Service) of the Lisbon Hospital Centre and Faculty of Medical Sciences, Lisbon, Portugal. Clinical data were recorded at baseline (immediately before start of therapy) and at the time of $\mathrm{NAb}$ analysis. These included age at disease onset, disease and treatment durations, relapse rates (i.e. number of relapses during the last 24 months), disease disability (the expanded disability status scale, EDSS) [10], and disease severity (the multiple sclerosis severity scale, MSSS) [11]. Tobacco smoking habits and concomitant medications were also recorded. In this study, all patients labelled smokers reported smoking at least five cigarettes per day. No patient was taking lipid-lowering agents.

NAbs analysis was requested when clinical evaluation revealed disease progression and/or the occurrence of one or more relapses during the last 2 years of therapy. Disease progression was defined as an increase of one point or more on the EDSS, and sustained for at least 6 months [12]. Male patients were excluded from the study because of the limited numbers available. Patients were treated with IFNbeta-1a 22 or $44 \mu$ g s.c. 3 times weekly (Rebif) or with IFN-beta-1b $250 \mu \mathrm{g}$ s.c. every other day (Betaferon). Patients treated with IFN-beta-1a $30 \mu \mathrm{g}$ once a week (Avonex) were excluded because of the lower potential of Avonex to induce NAbs compared with the other formulations [1, 2]. All patients were required to sign an informed consent form, and the local ethics committee approved this study.

\section{Laboratory measurements}

Patients included were clinically stable and analysed for NAbs $24 \mathrm{~h}$ after the last interferon injection. At sampling none of the patients had suffered from a relapse and none had been treated with steroids for at least 1 month. Patients were considered NAbs-positive if they had two consecutive NAbs-positive blood test results, determined with an interval of at least 6 months. NAbs were measured by an antiviral neutralisation cytopathic effect assay (CPA) (Biomonitor A/S, Copenhagen, Denmark). A sample was considered positive if it neutralised $>20 \%$ of added IFNbeta $(3 \mathrm{LU} / \mathrm{ml})$. This assay has been clinically validated and described in detail previously $[2,13]$.

Lipoproteins and inflammatory markers were analysed on the same blood samples at the Faculty of Medical Sciences, Lisbon, Portugal. Triglycerides, total cholesterol, HDL cholesterol, apolipoproteins A-I (apoA-I) and B (apoB) and uric acid levels were determined with enzymatic methods by using a Hitachi 911 autoanalyzer and commercial kits (Roche Diagnostic, Mannheim, Germany). Apolipoprotein E (apoE) was determined by electroimmunodiffusion (Sebia, Emery, France) and LDL-cholesterol by using the Friedwald formula [14]. Neopterin was measured by ELISA (IBL, Hamburg, Germany), leptin by radioimmunoassay (LINCOResearch, St. Charles, MO, USA), and ferritin with a chemiluminescent immunoassay (Abbott, Longford, Ireland). The plasma lipoprotein profiles were also analysed in all patients before the beginning of interferon therapy. ApoE polymorphism was examined by using an isoelectric focusing method, as described previously [15].

\section{Statistical analysis}

Patient demographic and clinical characteristics were described using mean values [standard deviations and medians (interquartile range)] for continuous variables. These characteristics were compared between NAbs response groups using two-tailed $t$ tests for continuous and logistic regression analysis and Fisher's exact tests for categorical data, respectively. Multiple logistic regression analysis was used to adjust smoking habit to the factors found to be significant in univariate analysis. Odds ratios (OR) and 95\% confidence intervals (CI) are presented and statistical significance was considered at the $5 \%$ level.

\section{Results}

As shown in Table 1, disease activity, severity and disability progression did not differ between NAbs-negative patients and those $(35 \%)$ who became NAbs-positive.

Concentrations of lipids and inflammatory markers, determined at the moment of NAbs analysis, are shown in Table 2. Compared with NAbs-positives, patients without NAbs had higher apoE OR 0.33 (95\% CI 0.11-0.96), $P=0.04$ and neopterin levels OR $0.19 \quad(95 \%$ CI 
Table 1 Clinical and demographic characteristics of female multiple sclerosis patients before the beginning of IFN-beta therapy and at the moment of NAb analysis

\begin{tabular}{|c|c|c|c|c|c|c|}
\hline & \multicolumn{3}{|c|}{ Status before treatment } & \multicolumn{3}{|c|}{ Status at the moment of NAb analysis } \\
\hline & $\begin{array}{l}\text { Remained } \\
\text { NAb-negative } \\
(n=20)\end{array}$ & $\begin{array}{l}\text { Became } \\
\text { NAb-positive } \\
(n=11)\end{array}$ & $P$ value & $\begin{array}{l}\text { NAb- } \\
\text { negative } \\
(n=20)\end{array}$ & $\begin{array}{l}\text { NAb- } \\
\text { positive } \\
(n=11)\end{array}$ & $P$ value \\
\hline Age: mean years (SD) & $39(9.8)$ & $42(9.3)$ & 0.38 & $44(10)$ & $47(9.5)$ & 0.41 \\
\hline Age at onset: mean years (SD) & $31(9.3)$ & $36(9.3)$ & 0.14 & & & \\
\hline Disease duration: mean years (SD) & $8.9(7.6)$ & $6.5(4.7)$ & 0.36 & $13.9(7.9)$ & $12(5.7)$ & 0.36 \\
\hline Treatment duration: mean years (SD) & & & & $5.05(1.9)$ & $4.9(2.8)$ & 0.87 \\
\hline Annual relapse rate: mean (SD) & $0.83(0.41)$ & $1.14(0.67)$ & 0.13 & $0.68(0.57)$ & $0.95(0.65)$ & 0.23 \\
\hline EDSS score: mean (SD) & $3.3(1.3)$ & $3.2(1.5)$ & 0.86 & $4.8(1.3)$ & $4.2(1.5)$ & 0.21 \\
\hline MSSS score: mean (SD) & $5.1(2.2)$ & $5.2(2.2)$ & 0.84 & & & \\
\hline Smokers: $n$ (\% of total $)$ & $6(30)$ & 7 (64) & 0.13 & $6(30)$ & 7 (64) & 0.13 \\
\hline Patients on other medications: $\mathrm{n}$ (\% of total) & $4(20)$ & $3(27)$ & 0.67 & $3(15)$ & $2(18)$ & 0.83 \\
\hline \multicolumn{7}{|l|}{ IFN-beta preparation } \\
\hline Rebif: $n$ (\% of total) & & & & $13(65)$ & $5(45)$ & 0.45 \\
\hline Betaferon: $n$ ( $\%$ of total) & & & & $7(35)$ & $6(54)$ & 0.45 \\
\hline
\end{tabular}

IFN interferon, $N A b$ neutralising antibody, SD standard deviation, EDSS expanded disability status scale, MSSS Multiple sclerosis severity scale $P$ values were calculated using two-sample $t$ test and Fisher's exact test

Table 2 Plasma lipoprotein profile and inflammatory markers in NAb-negative and -positive female multiple sclerosis patients

\begin{tabular}{|c|c|c|c|c|}
\hline & NAb-negative $(n=20)$ & NAb-positive $(n=11)$ & $\mathrm{OR}^{\mathrm{a}}$ & $P$ value \\
\hline Triglycerides (mg/dL) & $112(97-152)$ & $125(122-184)$ & $1.30(0.63,2.71)$ & 0.48 \\
\hline Total cholesterol (mg/dL) & $209(190-220)$ & $236(176-248)$ & $1.29(0.60,2.81)$ & 0.52 \\
\hline LDL-cholesterol (mg/dL) & $136(120-149)$ & $140(89-166)$ & $1.03(0.49,2.17)$ & 0.93 \\
\hline HDL-cholesterol (mg/dL) & $47(41-58)$ & $48(45-51)$ & $0.97(0.45,2.09)$ & 0.94 \\
\hline Apolipoprotein A-I (mg/dL) & $147(130-165)$ & $158(135-191)$ & $2.01(0.86,2.43)$ & 0.11 \\
\hline Apolipoprotein B (mg/dL) & $90(76,103)$ & $108(80-118)$ & $2.00(0.87,2.56)$ & 0.10 \\
\hline Apolipoprotein E (mg/L) & $76(66-87)$ & $53(50-84)$ & $0.33(0.11,0.96)$ & 0.04 \\
\hline Neopterin $(\mathrm{ng} / \mathrm{mL})$ & $12(9-14)$ & $7(4-10)$ & $0.19(0.05,0.76)$ & 0.02 \\
\hline Ferritin $(\mathrm{ng} / \mathrm{mL})$ & $70(26-223)$ & $60(30-70)$ & $0.40(0.13,1.25)$ & 0.12 \\
\hline Leptin (ng/mL) & $19(9-34)$ & $11(6-17)$ & $0.47(0.18,1.23)$ & 0.12 \\
\hline Uric acid (mg/dL) & $4.3(3.5-4.9)$ & $3.9(3.3-4.4)$ & $0.76(0.39,1.48)$ & 0.42 \\
\hline Body mass index $\left(\mathrm{kg} / \mathrm{m}^{2}\right)$ & $27(21-31)$ & $23(21-30)$ & $0.81(0.40,1.65)$ & 0.56 \\
\hline
\end{tabular}

$N A b$ neutralising antibody, $L D L$ low-density lipoprotein, $H D L$ high-density lipoprotein, $O R$ Odds ratio

${ }^{\text {a }}$ Odds of being NAbs positive relative to NAbs negative per 1-SD difference

Data are shown as medians with interquartile ranges. $P$ values were calculated using univariate logistic regression analysis

0.05-0.76), $P=0.02$. Before the beginning of therapy, patients who later remained NAbs-negative also had higher levels of apoE than those who became NAbs-positive [OR 0.32 (95\% CI 0.11-0.89), $P=0.03$ ]; Table 3. ApoE4 and apoE2 isoforms were present in 10 and $15 \%$ of patients without NAbs and in 18 and $9 \%$ of those who developed NAbs, $P=0.52$ and $P=0.64$, respectively.

When adjusting for age and smoking, for one-standard deviation decrease in apoE levels the odds of becoming NAb-positive increases 5.6-fold [OR 0.18 (95\% CI 0.04-
0.77), $P=0.04]$. When adjusting for apoE, smoking habit became significantly associated with NAbs production [OR 5.6, (95\% CI 1.3-86.9), $P=0.03$ ]. No associations were found between apoE and smoking and the studied inflammatory markers nor between relapse rates (before and after treatment) and apoE levels (data not shown). However, when dichotomizing apoE levels from its median value, levels $<74 \mathrm{mg} / \mathrm{L}$ appeared to be associated with higher relapse rates before treatment $(P=0.02)$, but not with relapse rates after treatment $(P=0.56)$. 
Table 3 Plasma lipoprotein profile in multiple sclerosis patients before the beginning of IFN-beta therapy

\begin{tabular}{|c|c|c|c|c|}
\hline & Remained NAb-negative $(n=20)$ & Became NAb-positive $(n=11)$ & $\mathrm{OR}^{\mathrm{a}}$ & $P$ value \\
\hline Triglycerides (mg/dL) & $113(67-135)$ & $94(83-147)$ & $1.47(0.68,3.20)$ & 0.33 \\
\hline Total cholesterol (mg/dL) & $200(170-228)$ & $213(193-228)$ & $1.31(0.76,2.27)$ & 0.38 \\
\hline LDL-cholesterol (mg/dL) & $126(108-149)$ & $136(117-160)$ & $1.24(0.59,2.60)$ & 0.58 \\
\hline HDL-cholesterol (mg/dL) & $56(49-60)$ & $52(42-67)$ & $0.85(0.39,1.86)$ & 0.68 \\
\hline Apolipoprotein A-I (mg/dL) & $157(141-168)$ & $155(134-166)$ & $0.88(0.41,1.88)$ & 0.74 \\
\hline Apolipoprotein B (mg/dL) & $96(87-119)$ & $107(88-118)$ & $1.51(0.70,3.27)$ & 0.29 \\
\hline Apolipoprotein E (mg/L) & $83(68-107)$ & $67(47-74)$ & $0.32(0.11,0.89)$ & 0.03 \\
\hline
\end{tabular}

For abbreviations, see Tables 1 and 2

Data are shown as medians (interquartile ranges). $P$ values were calculated using univariate logistic regression analysis

${ }^{\text {a }}$ Odds of being NAbs positive relative to NAbs negative per 1-SD difference

\section{Discussion}

Several studies have shown that the clinical efficacy of IFN-beta is reduced by the presence of NAbs (reviewed in Refs. $[1,2])$. However, a poor response to this treatment may also reflect the heterogeneity of the disease and individual genetic or metabolic differences not associated with the presence of NAbs $[12,16]$. Our cohorts of NAbspositive and -negative patients did not present significant differences in their clinical characteristics at baseline and at the moment of NAbs analysis. Hence, our findings suggest that innate differences in plasma apoE metabolism may be associated with the risk for NAbs development.

In addition to its well known role in lipid transport and metabolism, several studies have shown that apoE has immunosuppressive and anti-inflammatory effects (reviewed in Ref [6]). In MS, increased plasma apoE levels during exacerbations [17] could be related to a compensatory mechanism making use of the immunosuppressive effect of this apolipoprotein, as administration of apoEmimetic peptides attenuates inflammatory responses in murine models of the disease [18]. The observed association between lower apoE levels and the risk for NAbs production in our study supports this notion, as does the association between lower apoE levels and higher relapse rates found in patients only before the beginning of therapy and therefore independent of NAbs developed at later stages. Many studies have shown that the immune system directly influences lipoprotein homeostasis [5, 6, 19]. Inflammatory insults are associated with a decreased clearance and increased circulating apoE, which may promote its anti-inflammatory and immunomodulatory effects [19]. Hence, innate differences in apoE-containing lipoprotein metabolism might also influence NAb formation in MS patients in that an adaptive upregulatory response of plasma apoE to systemic inflammation might protect against NAbs development. This hypothesis is supported by data suggesting that MS patients who produce anti-IFNbeta binding antibodies have an increased incidence of autoreactive antibodies, including antiphospholipid and antithyroid antibodies [20]. The possible involvement of lipoprotein metabolism in NAbs production is of further interest in the context of other studies [7,8], implicating a disturbance of this metabolism in MS and the potential benefit of statin monotherapy in these patients [21].

Our results confirm previous work on an induction of serum neopterin levels by this treatment and a decrease of its levels in patients who develop NAbs [1]. Most interestingly, multivariate analysis suggests that smoking habits may be associated with NAbs induction in these patients. The mechanism(s) behind this is unknown. However, cigarette smoking is known to stimulate inflammatory responses and to promote the formation of autoantibodies in systemic autoimmune disorders [22]. Regardless of the underlying mechanisms, our data suggest that smoking habits should be evaluated in future studies of MS patients treated with IFN-beta biologics.

In addition to the small sample size and restriction to female gender, we recognize the limitations of the crosssectional design of our study. The associations suggested by our findings need confirmation in an expanded context of prospectively collected samples. In conclusion, this work suggests that the risk for NAbs production in MS patients treated with IFN-beta may be associated with apoE-containing lipoprotein metabolism and, possibly, with tobacco smoking. Further studies are needed to substantiate these findings, which could open new therapeutic and prophylactic strategies for this disease.

Acknowledgments We thank nurses Cristina Araújo and Ana Mendes, and Mrs Firmina Lebre and Augusta Marques for help in research. We are also grateful to BioEPI, Clinical and Translational Research Centre, for assistance in statistical analysis by Dr. Joana Vasconcelos and for epidemiological revision by Dr. Elizabeth 
Benito-Garcia. This work was supported by a grant from Biogen idec Portugal (RECONVERT project).

\section{References}

1. Hartung HP, Polman C, Bertolotto A et al (2007) Neutralising antibodies to interferon $\beta$ in multiple sclerosis: expert panel report. J Neurol 254:827-837

2. Bendtzen K, Kromminga A (2008) Immunogenicity of interferonbeta. In: van de Weert M, Moller EH (eds) Biotechnology: pharmaceutical aspects vol VIII. Immunogenicity of biopharmaceuticals. Springer, New York, pp 127-136

3. Hoffmann S, Cepok S, Grummel V et al (2008) HLADRB $1 * 0401$ and HLA-DRB $1 * 0408$ are strongly associated with the development of antibodies against interferon- $\beta$ therapy in multiple sclerosis. Am J Hum Genet 83:219-227

4. Gilli F, Valentino P, Caldano M (2008) Expression and regulation of IFNalpha/beta receptor in IFNbeta-treated patients with multiple sclerosis. Neurology 71:1940-1947

5. Lo JC, Wang Y, Tumanov AV et al (2007) Lymphotoxin alpha receptor-dependent control of lipid homeostasis. Science 316:285-288

6. Getz SG, Reardon CA (2009) Apoprotein E as a lipid transport and signalling protein in the blood, liver, and artery wall. J Lipid Res S156-S161

7. Giubilei F, Antonini G, De Legges S et al (2002) Blood cholesterol and MRI activity in first episode suggestive of multiple sclerosis. Acta Neurol Scand 106:109-112

8. Jamroz-Wisniewska A, Beltowski J, Stelmasiak Z, BartosikPsujek H (2009) Paraoxonase 1 activity in different types of multiple sclerosis. Mult Scler 15:399-402

9. McDonald WI, Compston A, Edan G et al (2001) Recommended diagnostic criteria for multiple sclerosis: guidelines from the international panel on diagnosis of multiple sclerosis. Ann Neurol 50:121-127

10. Kurtzke JF (1983) Rating neurological impairment in multiple sclerosis: an expanded disability status scale (EDSS). Neurology 33:1444-1452
11. Roxburgh RH, Seaman SR, Masterman T et al (2005) Multiple sclerosis severity score: using disability and disease duration to rate disease severity. Neurology 64:1144-1151

12. Rio J, Nos C, Tintoré $M$ et al (2006) Defining the response to interferon-beta in relapsing-remitting multiple sclerosis patients. Ann Neurol 59:344-352

13. Sorensen PS, Ross C, Clemmesen KM et al (2003) Clinical importance of neutralising antibodies against interferon beta in patients with relapsing-remitting multiple sclerosis. Lancet 362:1184-1191

14. Friedewald WT, Levy RI, Fredrickson DS (1972) Estimation of the concentration of low-density lipoprotein cholesterol in plasma, without use of the preparative ultracentrifuge. Clin Chem 18:499-502

15. Sena A, Couderc Ferret-SenaV et al (2009) Apolipoprotein E polymorphism interacts with cigarette smoking in progression of multiple sclerosis. Eur J Neurol 116:832-837

16. Comabella M, Lunemann JD, Rio J et al (2009) A type I interferon signature in monocytes associated with poor response to interferon-beta in multiple sclerosis. Brain 132:3353-3365

17. Carlsson J, Armstrong VW, Reiber H et al (1991) Clinical relevance of the quantification of apolipoprotein $\mathrm{E}$ in cerebrospinal fluid. Clin Chem Acta 196:167-176

18. Feng-Qiao L, Sempowski GD, McKenna SE et al (2006) Apolipoprotein E-derived peptides ameliorate clinical disability and inflammatory infiltrates into the spinal cord in murine model of multiple sclerosis. J Pharmacol Exp Ther 318:956-965

19. Li L, Thompson PA, Kitchens RL (2008) Infection induces a positive acute phase apolipoprotein $\mathrm{E}$ response from a negative acute phase gene: role of hepatic LDL receptors. J Lipid Res 49:1782-1793

20. Garg N, Weinstock-Guttman B, Bhasi K, Locke J, Ramanathan M (2007) An association between autoreactive antibodies and anti-interferon- $\beta$ antibodies in multiple sclerosis. Mult Scler 13:895-899

21. Sena A, Pedrosa R, Graça Morais M (2004) Statins for multiple sclerosis. Lancet 364:412

22. Sapori M (2002) Effects of cigarette smoke on the immune system. Nat Rev Immunol 2:372-377 